BURQUE et al.

REF-ISSN1808-0804 Vol.XII (1),11-26, 2015.

\title{
INTERAÇÕES MEDICAMENTOSAS VERIFICADAS EM UM GRUPO DE PACIENTES COM TRANSTORNO BIPOLAR
}

DRUG INTERACTIONS VERIFIED IN A GROUP OF PATIENTS WITH BIPOLAR DISORDER

INTERACCIONES MEDICAMENTOSAS VERIFICADAS EN UN GRUPO DE PACIENTES CON TRASTORNO BIPOLAR

\author{
Renan Kubiachi Burque ${ }^{1}$, Lenise Petter Francesconi ${ }^{1}$, André Tavares \\ Victorino ${ }^{1}$, Marcello Ávila Mascarenhas ${ }^{1}$, Keila Maria Ceresér ${ }^{1}$ \\ ${ }^{1}$ Centro Universitário Metodista - IPA, Porto Alegre, RS. Endereço: Rua Coronel Joaquim \\ Pedro Salgado, 80, Curso de Farmácia, CEP 90420-060, Porto Alegre, RS. \\ E-mail*: renan_kade2@hotmail.com
}

Submetido em: 13/11/2014; Aceito em: 16/04/2015

RESUMO: A farmacoterapia no Transtorno Bipolar (TB) visa diminuir a frequência e gravidade dos episódios. Nosso objetivo foi identificar interações medicamentosas em pacientes bipolares, através de um estudo transversal, em um hospital terciário. Foram identificados 150 pacientes e destes selecionamos 84 que eram polimedicados e/ou com comorbidades clínicas e/ou má-adesão, mas houve contato com apenas 51 e destes, 36 aceitaram participar. A coleta dos dados foi realizada pela análise do prontuário e entrevista. A classificação das interações considerou somente aquelas encontradas em pelo menos duas bibliografias idôneas, sendo estas classificadas conforme gravidade, e consideradas apenas as moderadas e graves. Identificamos 36 interações, sendo: entre não-psicofármacos (9), entre psicofármacos e não-psicofármacos (12), entre fármacos e álcool (5) e entre 
BURQUE et al.

REF-ISSN1808-0804 Vol.XII (1),11-26, 2015.

psicofármacos (10). A maior parte foi de gravidade moderada (31), porém 5 consideradas graves e destas, 3 desencadeiam cardiotoxicidade. Os pacientes utilizavam principalmente: estabilizadores de humor (32), antipsicóticos (26) e diuréticos (16). Nossos achados sugerem que as interações medicamentosas graves para os pacientes bipolares polimedicados podem desencadear outras complicações, como toxicidade cardíaca, associada à taquicardia. A identificação e monitoramento dos pacientes com transtorno bipolar poderá contribuir para uma melhora na conduta terapêutica e bem estar do paciente.

\section{PALAVRAS-CHAVE: Interações de Medicamentos. Transtorno Bipolar.} Atenção Farmacêutica.

ABSTRACT: Pharmacotherapy in Bipolar Disorder (BD) aims to reduce the frequency and severity of episodes. Our goal was to identify drug interactions in bipolar patients through a cross-sectional study in a tertiary hospital. We identified 150 patients in which 84 were selected, who were polymedicated and / or with comorbidities and / or bad adhesion. However, there was a contact with 51 of those patients and only 36 agreed to participate. Data collection was performed by analysis of medical records and interview. The classification of interactions only considered those which were found in at least two reputable bibliographies, and were classified according to severity, only considering the moderate and the severe ones. 36 interactions were identified, being: among non-psychiatric drugs (9), between non-psychoactive and psychotropic drugs (12), between drugs and alcohol (5) and among psychiatric drugs (10). Most were moderate severity (31), but five were considered severe, in which three triggered cardiotoxicity. The patients mainly used: mood stabilizers (32), antipsychotics (26) and diuretics (16). Our findings suggest that serious drug interactions for bipolar polymedicated patients can trigger other complications, such as heart toxicity associated with tachycardia. The identification and monitoring of patients with bipolar disorder may contribute to an improvement in the therapeutic conduct and well being of the patient.

\section{KEYWORDS: Drug Interactions. Bipolar Disorder. Pharmaceutical Care.}

RESUMEN: La farmacoterapia en el Trastorno Bipolar (TB) pretende disminuir la frecuencia y gravedad de los episodios. Nuestro objetivo fue identificar interacciones medicamentosas en pacientes bipolares a través de un estudio transversal en un hospital terciario. Identificamos 150 pacientes y de estos 


\section{BURQUE et al. \\ REF-ISSN1808-0804 Vol.XII (1),11-26, 2015.}

elegimos 84 que eran polimedicados y/o con enfermedades concomitantes clínicas y/o mala adhesión, pero hubo contacto con solamente 51 y de estos, 36 aceptaron participar. La recolección de datos fue realizada por el análisis del prontuario y entrevista. La clasificación de las interacciones ha considerado solamente aquellas halladas en por lo menos dos bibliografías idóneas, siendo estas clasificadas como gravedad y consideradas solamente las moderadas y graves. Identificamos 36 interacciones siendo: entre no psicofármacos (9), entre psicofármacos y no psicofármacos (12), entre fármaco y alcohol (5) y entre psicofármacos (10). La mayor parte fue de gravedad moderada (31), pero 5 consideradas graves y de estas 3 desencadenan cardiotoxicidad. Los pacientes utilizaban principalmente: estabilizadores de humor (32), antipsicóticos (26) y diuréticos (16). Nuestros hallados sugieren que las interacciones medicamentosas graves para los pacientes bipolares polimedicados pueden desencadenar otras complicaciones como toxicidad cardíaca asociada a taquicardia. La identificación y monitorización de los pacientes con trastorno bipolar podrá contribuir para una mejora en la conducta terapéutica y bien estar del paciente.

PALABRAS-CLAVE: Interacciones de Drogas. Trastorno Bipolar. Atención Farmacéutica.

\section{INTRODUÇÃo}

O Transtorno Bipolar (TB) é uma doença crônica e grave, que afeta $1 \%$ da população mundial e está associado a altos índices de suicídio e desemprego. A idade precisa do início da doença é difícil de definir, porém estima-se em 18 e 23,5 anos, sem diferença significativa entre os sexos e raça. O curso clínico do TB é crônico, geralmente caracterizado por episódios agudos (mania ou depressão), quando há uma exacerbação dos sintomas, e por períodos subsindrômicos e de remissão (eutimia) ${ }^{1}$.

O TB pode ser subdividido em duas entidades diagnósticas: TB tipo I (episódios depressivos maiores recorrentes com episódios maníacos) e TB tipo II (episódios depressivos maiores recorrentes com episódios hipomaníacos) ${ }^{2}$. Ambos apresentam sintomas que envolvem alterações no humor, cognição e comportamento. A intensidade dos sintomas é variável, acarretando prejuízos no desempenho 


\section{- Revista Eletrônica de Farmácia}

BURQUE et al.

REF-ISSN1808-0804 Vol.XII (1),11-26, 2015.

das atividades diárias e em sofrimento pessoal ${ }^{3}$.

Em vista da gravidade da doença, o tratamento farmacológico é fundamental e visa diminuir a frequência dos episódios, a gravidade e melhorar as consequências psicossociais. 0 sucesso do tratamento medicamentoso sofre influência direta da adesão ao tratamento, porém os pacientes com TB não conseguem visualizar com clareza esta importância, por isso a necessidade do reforço sobre as consequências da descontinuação da medicação e conscientização sobre a doença ${ }^{4}$.

O tratamento do TB é complexo e individualizado, pois depende de diversos fatores e seu objetivo principal é manter o paciente eutímico, ou seja, remitir as crises maníacas, depressivas e/ou mistas e acima de tudo prevenir a recorrência de novos episódios. Existem muitos medicamentos para o tratamento do $T B$, mas de uma forma geral se faz o uso de estabilizadores de humor $(\mathrm{EH})$, antidepressivos, antipsicóticos e, em alguns casos benzodiazepínicos. Os EH são utilizados em toda fase do tratamento, podendo ser utilizados como monoterapia, tanto na profilaxia da doença como nas fases agudas, principalmente na mania. Dentro deste grupo de psicofármacos os mais prescritos são: lítio, ácido valpróico e carbamazepina, existindo outros fármacos que são alternativos quando o paciente não se adapta aos EH clássicos, como lamotrigina, oxcarbazepina e gabapentina ${ }^{5}$.

Estudo realizado por Gazalle e colaboradores $(2007)^{6}$ demonstrou que $84 \%$ dos pacientes estavam usando mais do que uma medicação e $19 \%$ estavam usando três ou mais medicações. As combinações de fármacos mais utilizadas por estes pacientes foram: lítio + valproato $(17 \%)$; lítio + antipsicóticos (10\%); lítio + valproato + antipsicóticos $(9 \%) ;$ e antidepressivos + outros fármacos $(6 \%)$.

Sabe-se que o TB está associado a uma variedade de comorbidades não psiquiátricas; Kemp e colaboradores (2009) ${ }^{7}$ realizaram um estudo e observaram que as comorbidades clínicas afetavam principalmente o sistema respiratório, neurológico e músculo esquelético. Outros estudos mostram uma prevalência de doenças cardiovasculares, obesidade e diabetes mellitus ${ }^{8}$.

Em uma série de pacientes jovens com TB, as comorbidades 


\section{- Revista Eletrônica de Farmácia}

BURQUE et al.

REF-ISSN1808-0804 Vol.XII (1),11-26, 2015.

clínicas mais prevalentes foram: diabetes, doenças da tireóide, hipercolesterolemia, cardiopatias, hipertensão arterial e obesidade, sendo que esta apresenta prevalência elevada, principalmente devido aos efeitos colaterais das medicações ${ }^{9,10}$.

Interação medicamentosa pode ser conceituada como o resultado de uma interferência no efeito de um medicamento por outro medicamento, alimentos, bebidas ou por alguns agentes químicos ambientais ${ }^{11}$.

As interações medicamentosas estão entre as maiores causas de morbi-mortalidade e podem ocorrer através de vários mecanismos. O mecanismo primário inclui efeitos de fármacos no metabolismo hepático, incluindo o citocromo P450, enzimas ou efeitos na glicuronidação e nos transportadores de fármacos, glicoproteína-P ou na absorção; interações farmacodinâmicas também são importantes. Algumas substâncias em combinação podem ter ação sinérgica, resultando em toxicidade ${ }^{12}$.
As interações podem ser desejáveis ou benéficas, quando, por exemplo, dois fármacos são utilizados para aumentar a ação ou reduzir efeitos adversos de outro, ou indesejáveis, quando não levam a efeitos terapêuticos ou aumentam os efeitos adversos. Aquelas que resultam na redução da atividade de um fármaco podem ser difíceis de serem detectadas, podendo ser responsáveis pelo insucesso do tratamento e progressão da doença ${ }^{13}$.

Conforme Bachmann $(2006)^{14}$, as interações medicamentosas podem ser classificadas farmacologicamente quanto á gravidade, como: de menor gravidade (efeitos tolerados e sem necessidade de intervenção médica na maior parte dos casos), moderadas (intervenção médica é necessária) e graves (quando o efeito pode causar invalidez ou morte do paciente) $)^{13,14}$.

Sendo assim, este trabalho teve como objetivo identificar interações medicamentosas em um grupo de pacientes com transtorno bipolar.
Foi realizado no Hospital de Clínicas de Porto Alegre, um estudo 
BURQUE et al.

REF-ISSN1808-0804 Vol.XII (1),11-26, 2015.

descritivo transversal, com amostra por conveniência, sendo a população de pesquisa composta por pacientes atendidos no Ambulatório de Tratamento de Transtorno de Humor Bipolar (PROTAHBI) no ano de 2012. Por meio de um protocolo geral já preenchido pelos psiquiatras foram identificados 150 pacientes em atendimento no ambulatório e destes foram selecionados 84 pacientes que preenchiam os seguintes critérios de inclusão: polifarmácia e/ou comorbidades clínicas e/ou máadesão, eutimia no momento da inclusão, mas houve contato com apenas $60,71 \%(n=51)$ dos sujeitos; os demais se recusaram a participar do estudo por falta de tempo. A adesão referente aos medicamentos psiquiátricos foi avaliada através do auto-relato e dosagem sérica do estabilizador de humor. Depois de realizar o contato inicial, 36 pacientes aceitaram participar do estudo. Foram excluídos pacientes com retardo mental, em episódio grave e aqueles menores de 18 anos.

A coleta dos dados foi realizada por três pesquisadores devidamente treinados, após assinatura do termo de consentimento, onde através de análise do prontuário e entrevista foram coletadas as informações.
Para a classificação das interações foram consideradas somente aquelas encontradas em pelo menos duas das seguintes fontes: base de dados Micromedex ${ }^{15}$ e os livros Martindale ${ }^{16}$ ou Bachmann ${ }^{14}$. As interações foram classificadas de acordo com a base de dados Micromedex ${ }^{15}$ conforme sua gravidade, podendo ser leve, moderada ou grave, sendo incluídas apenas as interações classificadas como moderadas e graves.

O projeto foi aprovado pelo Comitê de Ética em Pesquisa do Hospital de Clínicas de Porto Alegre (Projeto $n^{\circ}$ 110210) e Comitê de Ética em Pesquisa do Centro Universitário Metodista, do IPA (Projeto no 405/2011).

Os dados foram analisados no Programa Statistical Package for Social Sciences (SPSS) versão 18.0, sendo descritos como frequências e média \pm desvio padrão, conforme classificação das variáveis.

\section{RESULTADOS E DISCUSSÃO}

Houve desistência de três pacientes entre os trinta e seis inicialmente incluídos no estudo. A tabela a seguir apresenta o perfil demográfico dos participantes do estudo. 
BURQUE et al.

REF-ISSN1808-0804 Vol.XII (1),11-26, 2015.

Tabela 1: Características sócio-demográficas e clínicas

\begin{tabular}{|c|c|}
\hline Variável & Casos $(n=33)$ \\
\hline \multicolumn{2}{|l|}{${ }^{\#}$ Sexo } \\
\hline Feminino & $25(75,8 \%)$ \\
\hline *Idade & $50,36 \pm 9,92$ \\
\hline \multicolumn{2}{|l|}{ \#Escolaridade } \\
\hline Fundamental incompleto & $7(21,2 \%)$ \\
\hline Fundamental completo & $6(18,2 \%)$ \\
\hline Médio incompleto & $4(12,1 \%)$ \\
\hline Médio completo & $5(15,2 \%)$ \\
\hline Superior incompleto & $5(15,2 \%)$ \\
\hline Superior completo & $6(18,2 \%)$ \\
\hline \multicolumn{2}{|l|}{ "Diagnóstico } \\
\hline Transtorno Bipolar tipo I & $24(72,7 \%)$ \\
\hline Transtorno Bipolar tipo II & $6(18,2 \%)$ \\
\hline Transtorno Bipolar SOE & $3(9,1 \%)$ \\
\hline \multicolumn{2}{|l|}{ \#Tentativa de suicídio } \\
\hline Sim & $22(66,7 \%)$ \\
\hline \multicolumn{2}{|l|}{ \#Consome café } \\
\hline Sim & $21(66,6 \%)$ \\
\hline \multicolumn{2}{|l|}{ \#Tabagismo atual } \\
\hline Sim & $12(33,4 \%)$ \\
\hline \multicolumn{2}{|l|}{ \#Alcoolismo atual } \\
\hline Sim & $10(69,7 \%)$ \\
\hline
\end{tabular}


BURQUE et al.

REF-ISSN1808-0804 Vol.XII (1),11-26, 2015.

\begin{tabular}{ll}
\hline${ }^{\#}$ Uso de drogas atual & \\
Sim & $1(3,0 \%)$
\end{tabular}

\section{\#Prática de atividade física}

Sim $9(27,3 \%)$

\section{\#Uso de vitaminas}

Sim $7(21,2 \%)$

Não respondeu

${ }^{\#}$ Alergia a medicamentos ou alimentos

Sim

$8(24,2 \%)$

${ }^{\#}=$ frequência relativa $\mathrm{n}$ (frequência relativa); * = média \pm desvio-padrão;

SOE $=$ sem outra especificação

O número de medicamentos utilizados variou entre três e treze, sendo que 11 pacientes $(33,3 \%)$ utilizavam entre três e cinco medicamentos; sendo também este percentual usuário de seis a oito medicamentos; 10 pacientes $(30,3 \%)$ utilizavam entre nove e onze medicamentos e um paciente utilizava treze medicamentos.

A quantidade de medicamentos aumenta conforme a progressão da doença e o número de comorbidades clínicas ${ }^{6}$.

Os medicamentos não-psiquiátricos mais utilizados pelos pacientes foram:

\section{$1(3,0 \%)$}

diuréticos e anti-hipertensivos $(63,6 \%)$, hormônios da tireoide e análogos $(36,4 \%)$, hipoglicemiantes orais $(36,4 \%)$ e agentes antilipêmicos $(30,3 \%)$. Os psicofármacos mais utilizados foram: ácido valproico/valproato de sódio $(51,5 \%)$, carbonato de lítio $(36,4 \%)$, risperidona $(36,4 \%)$, clorpromazina $(24,2 \%)$ e clonazepam (30,3\%).

Foram consideradas somente as interações medicamentosas encontradas em pelo menos duas das seguintes fontes: Cordioli ${ }^{17}$, Bachmann ${ }^{14}$, Martindale ${ }^{16}$ e Micromedex ${ }^{15}$. 
BURQUE et al.

REF-ISSN1808-0804 Vol.XII (1),11-26, 2015.

As interações medicamentosas identificadas encontram-se descritas nas tabelas $2,3,4$ e 5 .

Tabela 2: Interações entre não-psicofármacos verificadas em um grupo de 33 pacientes com transtorno bipolar

\begin{tabular}{|c|c|c|c|}
\hline Fármacos & Gravidade & Efeito & Ocorrência (f) \\
\hline $\begin{array}{l}\text { Hipoglicemiantes orais } \\
\text { e hidroclorotiazida }\end{array}$ & Moderada & $\begin{array}{l}\text { *Poderá ocorrer hipo ou } \\
\text { hiperglicemia. }\end{array}$ & 6 \\
\hline $\begin{array}{l}\text { IECA ou losartano ou } \\
\text { hidroclorotiazida } x \\
\text { AINEs }\end{array}$ & Moderada & $\begin{array}{l}\text { *Poderá ocorrer redução do } \\
\text { efeito anti-hipertensivo. }\end{array}$ & 5 \\
\hline $\begin{array}{l}\text { Hipoglicemiantes orais } \\
\text { e beta-bloqueadores }\end{array}$ & Moderada & $\begin{array}{l}\text { *Poderá ocorrer hipo ou } \\
\text { hiperglicemia. }\end{array}$ & 4 \\
\hline $\begin{array}{l}\text { Levotiroxina } \\
\text { e propranolol }\end{array}$ & Moderada & $\begin{array}{l}\text { *O propranolol poderá reduzir } \\
\text { os níveis séricos de T3. }\end{array}$ & 4 \\
\hline $\begin{array}{l}\text { Salbutamol } \\
\text { ou xantinas } \\
\text { e beta-bloqueadores }\end{array}$ & Moderada & $\begin{array}{l}\text { * o propranolol poderá } \\
\text { antagonizar a ação } \\
\text { broncodilatadora do } \\
\text { salbutamol ou das xantinas. }\end{array}$ & 4 \\
\hline $\begin{array}{l}\text { Insulina } \\
\text { e beta-bloqueadores }\end{array}$ & Moderada & $\begin{array}{l}\text { *Poderá ocorrer bloqueio dos } \\
\text { sintomas de hipoglicemia pelo } \\
\text { bloqueio beta-adrenérgico. }\end{array}$ & 3 \\
\hline $\begin{array}{l}\text { Propranolol } \\
\text { e xantinas }\end{array}$ & Moderada & $\begin{array}{l}\text { *O propranolol poderá } \\
\text { aumentar os níveis séricos } \\
\text { das xantinas por inibir sua } \\
\text { eliminação; por outro lado, } \\
\text { poderá antagonizar a ação } \\
\text { broncodilatadora destas. }\end{array}$ & 1 \\
\hline $\begin{array}{l}\text { Alopurinol } \\
\text { e hidroclorotiazida }\end{array}$ & Moderada & $\begin{array}{l}\text { *A hidroclorotiazida poderá } \\
\text { aumentos dos níveis de ácido } \\
\text { úrico, especialmente em } \\
\text { pacientes com disfunção } \\
\text { renal. }\end{array}$ & 1 \\
\hline $\begin{array}{l}\text { Prednisona } \\
\text { e ciclosporina }\end{array}$ & Moderada & $\begin{array}{l}\text { *A prednisona e outros } \\
\text { fármacos da classe poderão } \\
\text { aumentar a concentração } \\
\text { sérica da ciclosporina e vice- }\end{array}$ & 1 \\
\hline
\end{tabular}


BURQUE et al.

REF-ISSN1808-0804 Vol.XII (1),11-26, 2015.

\section{versa.}

$\mathrm{f}=$ frequência absoluta; IECA=Inibidores da Enzima Conversora da Angiotensina;

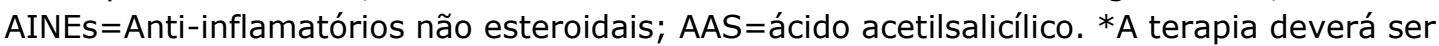
monitorizada.

Tabela 3: Interações entre psicofármacos e não-psicofármacos verificadas em um grupo de 33 pacientes com transtorno bipolar

\begin{tabular}{|c|c|c|c|}
\hline Fármacos & Gravidade & Efeito & Ocorrência (f) \\
\hline $\begin{array}{l}\text { Benzodiazepínicos } \\
\text { e anti-hipertensivos } \\
\text { ou diuréticos } \\
\text { (tiazídicos e de alça) }\end{array}$ & $\begin{array}{l}\text { Moderada com } \\
\text { bloqueadores dos } \\
\text { canais de cálcio e } \\
\text { com demais, baixa }\end{array}$ & $\begin{array}{l}\text { "Poderá ocorrer aumento } \\
\text { dos níveis séricos dos } \\
\text { benzodiazepínicos e } \\
\text { ocorrer hipotensão } \\
\text { postural. }\end{array}$ & 5 \\
\hline Valproato e AAS & Moderada & $\begin{array}{l}\text { *Poderá ocorrer aumento } \\
\text { dos níveis séricos do } \\
\text { valproato. }\end{array}$ & 5 \\
\hline Lítio e IECA & Moderada & $\begin{array}{l}\text { \#Poderá ocorrer aumento } \\
\text { dos níveis séricos do lítio. }\end{array}$ & 3 \\
\hline Lítio e cafeína & Moderada & $\begin{array}{l}\text { \#A cafeína aumenta a } \\
\text { excreção renal do lítio. }\end{array}$ & 3 \\
\hline $\begin{array}{l}\text { Fenotiazínicos } \\
\text { e beta-bloqueadores }\end{array}$ & Moderada & $\begin{array}{l}\text { *Poderá ocorrer aumento } \\
\text { dos efeitos } \\
\text { extrapiramidais. }\end{array}$ & 3 \\
\hline $\begin{array}{l}\text { Fenotiazínicos } \\
\text { e hipoglicemiantes } \\
\text { orais }\end{array}$ & Moderada & $\begin{array}{l}\text { *Poderá ocorrer } \\
\text { hipoglicemia. }\end{array}$ & 3 \\
\hline $\begin{array}{l}\text { Benzodiazepínicos } \\
\text { e nicotina }\end{array}$ & Moderada & $\begin{array}{l}\text { **A nicotina aumenta a } \\
\text { metabolização dos } \\
\text { benzodiazepínicos. }\end{array}$ & 2 \\
\hline $\begin{array}{l}\text { Clozapina e } \\
\text { anti-hipertensivos }\end{array}$ & Maior & $\begin{array}{l}\text { *Poderá ocorrer } \\
\text { cardiotoxicidade. }\end{array}$ & 2 \\
\hline $\begin{array}{l}\text { Risperidona e beta- } \\
\text { bloqueadores }\end{array}$ & Moderada & $\begin{array}{l}\text { *Poderá ocorrer } \\
\text { hipotensão. }\end{array}$ & 2 \\
\hline
\end{tabular}


BURQUE et al.

REF-ISSN1808-0804 Vol.XII (1),11-26, 2015.

\begin{tabular}{llll}
\hline $\begin{array}{l}\text { Risperidona } \\
\text { e hidroclorotiazida }\end{array}$ & Moderada & $\begin{array}{l}\text { *Poderá ocorrer } \\
\text { hipotensão. }\end{array}$ & 1 \\
Simpaticomiméticos & Moderada & $\begin{array}{l}\text { *Poderá ocorrer } \\
\text { diminuição do } \\
\text { peristaltismo intestinal. }\end{array}$ & 1 \\
e amitriptilina & Maior & $\begin{array}{l}\text { *Poderá ocorrer } \\
\text { cardiotoxicidade. }\end{array}$ & 1 \\
e anti-hipertensivos & & & \\
\hline
\end{tabular}

$\mathrm{f}=$ frequência absoluta; IECA=Inibidores da Enzima Conversora da Angiotensina; AINEs=Antiinflamatórios não esteroidais; AAS=ácido acetilsalicílico. ${ }^{*}$ Monitorizar e considerar modificação de terapia. *A terapia deverá ser monitorizada. ** Ver a possibilidade do paciente parar de fumar.

Tabela 4: Interações entre fármacos e álcool verificadas em um grupo de 33 pacientes com transtorno bipolar

\begin{tabular}{|c|c|c|c|}
\hline Fármacos & Gravidade & Efeito & Ocorrência (f) \\
\hline Valproato & Moderada & $\begin{array}{l}\text { *Poderá ocorrer aumento } \\
\text { da depressão sobre o } \\
\text { Sistema Nervoso Central. }\end{array}$ & 3 \\
\hline Fluoxetina & Moderada & $\begin{array}{l}\text { *Poderá ocorrer aumento } \\
\text { da depressão sobre o } \\
\text { Sistema Nervoso Central. }\end{array}$ & 2 \\
\hline Risperidona & Moderada & $\begin{array}{l}\text { *Poderá ocorrer aumento } \\
\text { da depressão sobre o } \\
\text { Sistema Nervoso Central. }\end{array}$ & 2 \\
\hline Sulpirida & Moderada & $\begin{array}{l}\text { *Poderá ocorrer aumento } \\
\text { da depressão sobre o } \\
\text { Sistema Nervoso Central. }\end{array}$ & 1 \\
\hline Metformina & Moderada & $\begin{array}{l}\text { *Ocorrerá aumento do risco } \\
\text { de ocorrer acidose } \\
\text { metabólica. }\end{array}$ & 1 \\
\hline
\end{tabular}

$f=$ frequência absoluta. $*$ Evitar. 
BURQUE et al.

REF-ISSN1808-0804 Vol.XII (1),11-26, 2015.

Tabela 5: Interações entre psicofármacos verificadas em um grupo de 33 pacientes com transtorno bipolar

\begin{tabular}{|c|c|c|c|}
\hline Fármacos & Gravidade & Efeito & Ocorrência (f) \\
\hline Lítio e clorpromazina & Maior & $\begin{array}{l}\text { *Poderá ocorrer aumento } \\
\text { dos efeitos extrapiramidais } \\
\text { da clorpromazina. }\end{array}$ & 5 \\
\hline Lítio e sertralina & Moderada & $\begin{array}{l}\text { **Poderá ocorrer aumento } \\
\text { das concentrações séricas } \\
\text { do lítio. }\end{array}$ & 2 \\
\hline Valproato e clonazepam & Moderada & $\begin{array}{l}\text { *Poderá ocorrer } \\
\text { potencialização dos efeitos } \\
\text { sedativos dos } \\
\text { benzodiazepínicos; em } \\
\text { especial com o clonazepam, } \\
\text { poderão ocorrer crises de } \\
\text { ausência. }\end{array}$ & 2 \\
\hline $\begin{array}{l}\text { Valproato e } \\
\text { carbamazepina }\end{array}$ & Moderada & $\begin{array}{l}\text { *A carbamazepina poderá } \\
\text { aumentar o metabolismo do } \\
\text { valproato e este poderá } \\
\text { diminuir os níveis séricos da } \\
\text { carbamazepina. }\end{array}$ & 1 \\
\hline Valproato e primidona & Moderada & $\begin{array}{l}\text { *O valproato poderá } \\
\text { diminuir o metabolismo da } \\
\text { primidona. }\end{array}$ & 1 \\
\hline Fluoxetina e clonazepam & Moderada & $\begin{array}{l}\text { *A fluoxetina poderá } \\
\text { diminuir o metabolismo dos } \\
\text { benzodiazepínicos. }\end{array}$ & 1 \\
\hline Fluoxetina e codeína & Moderada & $\begin{array}{l}\text { *Poderá ocorrer síndrome } \\
\text { serotninérgica causada. }\end{array}$ & 1 \\
\hline $\begin{array}{l}\text { Fluoxetina e } \\
\text { clorpromazina }\end{array}$ & Maior & $\begin{array}{l}\text { *Poderá ocorrer aumento da } \\
\text { cardiotoxicidade. }\end{array}$ & 1 \\
\hline $\begin{array}{l}\text { Antidepressivos } \\
\text { tricíclicos e risperidona }\end{array}$ & Moderada & $\begin{array}{l}\text { *Os antidepressivos } \\
\text { tricíclicos poderão diminuir o } \\
\text { metabolismo da risperidona. }\end{array}$ & 1 \\
\hline $\begin{array}{l}\text { Codeína } \\
\text { e anticonvulsivantes }\end{array}$ & Maior & $\begin{array}{l}\text { *Poderá ocorrer depressão } \\
\text { respiratória. }\end{array}$ & 1 \\
\hline
\end{tabular}




\section{- Revista Eletrônica de Farmácia}

BURQUE et al.

REF-ISSN1808-0804 Vol.XII (1),11-26, 2015.

$\mathrm{f}=$ frequência absoluta. $*$ A terapia deverá ser monitorizada. $* *$ Monitorizar a litemia e considerar modificação de terapia.

As interações de maior prevalência ou maior gravidade, encontradas em nosso estudo, serão discutidas a seguir.

Alguns estudos não randomizados encontraram associação entre uso de diuréticos tiazídicos e hiperglicemia, enquanto que em outros tal associação não foi demonstrada ${ }^{18}$. A interação entre hidroclorotiazida e hipoglicemiantes orais foi observada em cinco pacientes do nosso estudo e foi recomendado a eles que não administrassem concomitantemente estas medicações e que ficassem atentos a sinais de hipoglicemia ou hiperglicemia, realizando também controle da dieta e exames periódicos.

Os anti-inflamatórios nãoesteroidais podem ocasionar um aumento da pressão arterial, principalmente em pessoas com história de hipertensão ou pacientes com insuficiência renal; com isso pode haver redução do efeitos de antihipertensivos como: inibidores da enzima conversora da angiotensina, losartano ou hidroclorotiazida; também esta associação pode aumentar a predisposição á insuficiência renal ${ }^{19}$. Esta interação foi observada em cinco pacientes do nosso estudo e foi recomendado monitoramento da pressão arterial e da função renal.

O uso de benzodiazepínicos e anti-hipertensivos ou diuréticos (tiazídicos e de alça) poderá acarretar em aumento dos níveis séricos dos benzodiazepínicos, especialmente com anti-hipertensivos bloqueadores dos canais de cálcio e com betabloqueadores; ainda com diuréticos e também com antihipertensivos, pode ocorrer hipotensão postural ${ }^{14}$. Cinco pacientes de nosso estudo podem ser suscetíveis a esta interação e foi recomendado monitoramento da pressão arterial e atenção aos sinais como tonturas e maior sedação.

Antipsicóticos atípicos, como a clozapina e a quetiapina, podem exacerbar a hipotensão com o uso de anti-hipertensivos; além disso, apresentam potencial cardiotóxico (miocardite e arritmias cardíacas) ${ }^{17}$. Três pacientes de nosso estudo podem ser suscetíveis a esta interação de maior intensidade e foi 
BURQUE et al.

REF-ISSN1808-0804 Vol.XII (1),11-26, 2015.

recomendado que mantivessem as consultas periódicas ao cardiologista.

O ácido acetilsalicílico aumenta os níveis séricos de valproato, por competição e deslocamento do valproato das proteínas plasmáticas, ou por inibição da beta-oxidação do valproato pelo ácido acetilsalicílico ${ }^{20}$, podendo-se esperar aumento da toxicidade, como: sonolência, confusão mental, náuseas e vômitos ${ }^{17}$. Esta interação foi observada em cinco pacientes do nosso estudo e, além do monitoramento frequente dos níveis

\section{CONCLUSÃO}

O elevado número de medicamentos utilizado pelos participantes deste estudo predispõe a ocorrência de interações medicamentosas.

Fatores genéticos, sexo, comorbidades, fatores ambientais e hábitos alimentares dos pacientes podem influenciar a ocorrência de interações medicamentosas. Normalmente pacientes com doenças crônicas são mais suscetíveis a interações medicamentosas.

A prevenção das interações medicamentosas é de suma séricos de valproato, os pacientes foram instruídos a estarem atentos aos sinais de toxicidade.

O lítio associado à clorpromazina pode causar aumento dos efeitos extrapiramidais, como: rigidez, disartria, estupor, discinesia; apesar da possível exacerbação da neurotoxicidade, esta combinação é muito utilizada no transtorno bipolar $^{17}$. Cinco pacientes de nosso estudo podem ser suscetíveis a esta interação, de elevada intensidade e a qualquer sinal de toxicidade, deverão contatar o seu médico.

importância para o sucesso do tratamento. Isso pode ser feito mediante conhecimento da história do paciente quanto a medicações utilizadas no passado e atualmente, bem como com aconselhamento quanto ao uso correto das medicações e reconhecimento de sinais de toxicidade ou de sub-efeitos. Antes do início do tratamento medicamentoso, devem ser solicitados aos pacientes exames laboratoriais, sendo estes repetidos durante o tratamento.

\section{AGRADECIMENTOS}

Agradecemos ao FIPE-HCPA por ter apoiado este trabalho e ao CNPq pela concessão de bolsa de 
BURQUE et al.

REF-ISSN1808-0804 Vol.XII (1),11-26, 2015.

Iniciação Científica ao primeiro autor deste artigo, que é parte do seu
Trabalho de Conclusão de Curso de Farmácia.

\section{REFERÊNCIAS}

Yatham L, Kennedy S, Schaffer A, Parikh S, Beaulieu S, O'Donovan C et al. Canadian Network for Mood and Anxiety Treatments (CANMAT) and International Society for Bipolar Disorders (ISBD) collaborative update of CANMAT guidelines for the management of patients with bipolar disorder: updated. Bipolar Disord. 2009;11(3):225-55.

American Psychiatric Association (APA): Diagnostic and Statistical Manual of Mental Disorders. Tradução de Maria Cristina Monteiro. Porto Alegre: ARTMED; 2003.p.172-179.

Vieta E, Colom F, Martínez-Aran A, Benabarre A, Gastó C. Personality disorders in bipolar II patients. J Nerv Ment Dis. 1999;187(4):245-8.

Velligan DI, Weiden PJ, Sajatovic M, Scott J, Carpenter D, Ross R et al. Expert consensus panel on adherence problems in serious and persistent mental illness. The expert consensus guideline series: adherence problems in patients with serious and persistent mental illness. J Clin Psychiatry. 2009;70(4):1-46.

Seddon K, Nutt D. Pharmacological treatment of bipolar disorder. Psychiatry. 2007;6(7):305-8.

Gazalle FK, Hallal PC, Tramontina J, Rosa AR, Andreazza AC, Zanatto V et al. Polypharmacy and suicide attempts in bipolar disorder. Rev Bras Psiquiatr. 2007;29(1):35-8.

Kemp DE, Gao K, Ganocy SJ, Emily C, Feldman K, Chan PK et al. Medical and substance use comorbidity in bipolar disorder. J Affect Disord. 2009;116(1-2):64-9.

Kupfer DJ. The increasing medical burden in bipolar disorder. JAMA. $2005 ; 293(20): 2528-30$.

McElroy SL, Kotwal R, Keck PE, Akiskal HS. Comorbidity of bipolar and eating disorders: distinct or related disorders with shared dysregulations. J Affect Disord. 2005;86(2):107-27. 
BURQUE et al.

REF-ISSN1808-0804 Vol.XII (1),11-26, 2015.

Goldstein B, Liu SM, Zivkovic N, Schaffer A, Chie LC, Blanco C. The burden of obesity among adults with bipolar disorder in the United States. Bipolar Disord. 2011;13(4)387-95.

Santos HC, Ribeiro RR, Ferrarini M, Fernandes JPS. Possíveis interações medicamentosas $\mathrm{cm}$ psicotrópicos encontradas em pacientes da Zona Leste de São Paulo. Revista de Ciências Farmacêuticas Básica e Aplicada. 2009;30(3):285-89.

McCance-Katz EF, Sullivan LE, Nallani S. Drug interactions of clinical importance among the opioids, methadone and buprenorphine, and other frequently prescribed medications: a review. Am J Addict. 2010;19(1):4-16.

Ditadi AC, Colet C. Interações Medicamentosas Potenciais em Ambiente Hospitalar: Uma revisão bibliográfica. Revista Contexto \& Saúde. 2010;10(18):29-36.

Bachmann KA. Interações Medicamentosas. 2. ed. São Paulo: Manole; 2006.890p.

Micromedex Healthcare Series. Greenwood Village, Colorado, consultado via Portal CAPES em Outubro de 2012.

Martindale. The Complete Drug Reference. 35 ed. London: The Royal Pharmaceutical Society; 2007.

Cordioli AV (organizador) et al. Psicofármacos. Consulta Rápida. 3 ed. Porto Alegre: Artmed; 2005.695p.

Sigel D, Saliba P, Haffner S. Glucose and insulin levels during diuretic therapy in hypertensive men. Hypertension. 1994;23(6):688-94.

Fournier JP, Sommet A, Durrieu G, Poutran JC, Lapeyre-Mestre M, Montastruc JL. French Network of Regional Pharmacovigilance Centres. Drug interactions between antihypertensive drugs and non-steroidal anti-inflammatory agents: a descriptive study using the French Pharmacovigilance database. Fundam Clin Pharmacol. $2014 ; 28(2): 230-5$.

De Leon J, Kiesel JL, Fleming MW, Strobl B. Valproic acid toxicity associated with low dose of aspirin and low total valproic acid levels: a case report. J Clin Psychopharmacol. 2009;29(5):509-11. 